\title{
COMMISSION 25: STELLAR PHOTOMETRY AND POLARIMETRY
}

(PHOTOMETRIE ET POLARIMETRIE STELLAIRE)

\author{
PRESIDENT: C. Sterken \\ VICE-PRESIDENT: A.U. Landolt \\ ORGANIZING COMMITTEE: M. Bessell, M. Breger, I. Glass, J. Graham, \\ H. Hensberge, D. Kurtz, J. Landstreet, E.F. Milone, T. Moffett, K. Sekiguchi, \\ J. Tinbergen \& W.H. Warren
}

The work of Commission 25 covers a wide range of topics concerning the measurement of magnitude, colour and polarisation of astronomical objects. As such, the area of interest covers virtually every field of astrophysical research in the visual and infrared spectral domain. Our reports cover some aspects of photometry and polarimetry as a technique rather than being an account of research highlights over the last three years.

As was already stressed in the previous Reports, a major problem remains the increasing decommissioning of photometric telescopes. At a time when CCD detectors become of so widespread use and allow observation to very deep magnitude, there is now more than ever a need for the establishment of consistent policies for instrument calibration, observation, data reduction and standardisation. A very clear message in this respect has been formulated in 1996 by participants of a meeting on photometry in Lithuania, and their resolution is included in this report. I also refer to E.F. Milone's report on the Working Group on Infrared Astronomy.

\section{PHOTOMETRY (C. Sterken)}

\subsection{Photometric Systems and synthetic photometry}

New photometric systems, mainly for use with CCDs are being proposed, and existing photomultiplier-based systems are being upgraded or extended for applications with CCD detectors. Maitzen et al. (1997, A\&A 327, 636) apply, for the first time, CCD-photometry in the three-filter $\Delta a$ system for chemically-peculiar stars, and conclude that the performance of their photometry perfectly matches the requirements of the conventional photometric technique. Liu \& Green (1998, AJ 116 1074) describe an optical (six broad-bandpass) multi-color system for measuring galaxy redshifts.

The Stromvil system is the addition to the Strömgren four-color photometric system of three Vilnius passbands at 374,516 and $656 \mathrm{~nm}$ that leads to a more universal photometric system allowing spectral classification over a broader range of spectral types. A preliminary calibration of the system in terms of spectral and luminosity classes, temperatures and surface gravities is available, and a list of preliminary standards is given by Straižys et al. (1996, BaltA 5, 83).

A brand new photometric system to be used in space is being designed by the GAIA photometry team. Heavily relying on expert advice of Strömgren, Vilnius and Geneva photometrists (Copenhagen, Vilnius and Geneva Observatories) supplemented by detailed modelling (Padua and Barcelona), this new system will be optimised in a way never seen before in the design of a new photometric system and will lead to photometric classification of $\mathrm{B}$ to $\mathrm{M}$ and $\mathrm{C}$ stars of all metallicities at various degrees of interstellar reddening. 
Castelli (1999, A\&A 346, 564) computed several grids of synthetic Johnson $U B V$ color indices based on ATLAS9 atmospheric models and investigated the dependence of the color indices on $T_{\text {eff }}, \log g$, metallicity, and microturbulent velocity.

\subsection{Photometric instruments}

Bless et al. (1999, PASP 111, 346) present an overview of the performance of the High Speed Photometer on the Hubble Space Telescope. The HSP was designed for precise high time-resolution photometry from visual to ultraviolet wavelengths, and it met or exceeded operational specifications, though the ability to carry out its primary program was severely hampered by the spherical aberration of the primary mirror. Graham (1998, PASP 110, 732) details VIRIS, a combined CCD+NICMOS3 system for near-simultaneous imaging at optical and near-infrared wavelengths ( 0.5 to $2.2 \mu \mathrm{m})$.

Henry (1999, PASP 111, 845) describes the operation of two automatic photoelectric telescopes and the quality-control techniques that result in routine acquisition of single-star high-precision differential photometry.

\subsection{Calibrations and transformations}

Gilliland et al. (1999, PASP 111, 1009) explored on-orbit data characteristics of the STISCCD aboard the HST. STIS data show excellent linearity at high count levels, even for extreme oversaturation of individual central pixels. At low count levels they interpret a position- and intensity-dependent nonlinearity in terms of charge transfer (in)efficiency in the parallel clocking direction and provide a simple model that accounts for this. Fabricius (1997, ESASP 402, 131) re-calibrated Guide Star Catalog magnitudes using the Tycho Catalogue as a photometric reference catalogue, and states that it is possible to bring the systematic errors of the GSC well below $0.1 \mathrm{mag}$ (for stars brighter than $11.5 \mathrm{mag}$ ). Stetson (1998, PASP 110, 1448) investigates charge-transfer effects in photometry with WFPC2 aboard HST by a comparison of WFPC2 observations with ground-based photometry for the Galactic globular clusters $\omega$ Centauri and NGC2419 and presents simple numerical formulae describing the fraction of lost signal as functions of position on the detector, stellar brightness, and the diffuse sky brightness recorded in an image.

Smith et al. (1997, AAS 191, 1612) present plans for photometric calibration of the Sloan Digital Sky Survey to be conducted at a 24-inch Monitor Telescope. This telescope will define the SDSS color system with a set of more than 100 standard stars, it will monitor extinction in each of the five survey filters on an hourly basis, and it will also track changes in the instrumental zeropoint by directly calibrating about $3 \%$ of the total survey area.

Harmanec $(1998, A \& A 335,173)$ gives a reliable transformation of Hipparcos $H_{p}$ magnitudes into Johnson $V$ and $B$ magnitudes. Straižys and co-workers present several papers with transformation equations between the standard and CCD Vilnius systems (see, for example, Straižys et al. 1997, BaltA 6, 445).

Manfroid (1996, A\&AS 118, 391) reports on numerical simulations of a CCD flatfielding calibration procedure on stellar fields in the monochromatic case, and shows how and why one must establish high-quality standard sequences in clusters or moderatelycrowded fields. Wild (1997, PASP 109, 1269) derives a functional equation that enables a flat-field frame to be generated that is independent of the illumination source (and its variations). Zhou et al. (1999, PASP 111, 909) present a method to self-calibrate the spectral energy distribution of objects in a survey based on the fitting of a databank of observed multicolor photometry. The self-calibration technique can improve the quality of observations that have not been taken under perfect photometric conditions.

Gray $(1998$, AJ 116, 482) publishes an improved absolute flux calibration for Strömgren $u v b y$ photometry tied to Taylor's system of critically selected spectrophotometric standards. 


\subsection{Standards stars}

Clausen et al. (1997, A\&AS 122, 559) list accurate standard uvby indices for 73 southern B, $A, F$ and $G$ stars with $V=8.2-10.9$ and covering all three transformation regions of the $u v b y$ system. Standard $\beta$ indices are included for the $55 \mathrm{~B}, \mathrm{~A}$, and $\mathrm{F}$ stars in the sample. This is a useful set of secondary standards for $u v b y \beta$ CCD photometry with southern hemisphere 1-2 $\mathrm{m}$ class telescopes. Cousins \& Caldwell (1996, MNRAS 281, 522) made observations of DDO equatorial standard stars, made with the same photometer and DDO filters as were used for the E-region stars in Cousins' earlier paper. Though there is a fair overall agreement between both sets of results, there remain some inevitable small systematic differences. Oja \& Evans $(1998$, A\&A 333, 673O) use photometric data from the TYCHO experiment of the HIPPARCOS mission to study discrepancies in $B-V$ between the different series of measurement of the equatorial $U B V$ standards and provide corrections to data of Landolt and Menzies et al.

Hammersley et al. $(1998$, A\&AS 128,207$)$ present $N, 8.7 \mu \mathrm{m}, 9.8 \mu \mathrm{m}, 12.5 \mu \mathrm{m}$ and $Q$ bolometry of about two dozen single, non-variable $K$ giants to serve as a new homogeneous set of mid IR standards. Hunt et al. (1998, AJ 115, 2594) report $J H K$ photometry of 86 stars in 40 uniformly-distributed Northern Hemisphere fields, thus providing a homogeneous broadband calibration network for near-infrared panoramic detectors. Persson et al. (1998, AJ 116, 2475) present a new grid of 65 faint near-infrared $J H K$ standard stars $(K=10-$ 12). Mueller \& Lagerros (1998, A\&A 338, 340) use asteroids as far-IR calibrators for the imaging photopolarimeter ISOPHOT on board of ISO.

\subsection{Catalogs and data bases}

van Leeuwen et al. (1997, A\&A 323, 61) give an overview of the Hipparcos and Tycho photometric data, with reference to the main aspects of the data reductions and the overall quality and quantity of the results. The data are conveniently presented in three forms: as mean values in the astrometric catalogues, as epoch photometry, and as interpreted results of the variability analysis. A detailed report on the astrometry and photometry from Tycho has been made by $\mathrm{E}$. $\mathrm{H} \emptyset \mathrm{g}$ and is included in the report of IAU Commission 8 .

Hall et al. (1996, ApJS 104, 185) assembled a catalog of more than 20000 stellar objects detected in six 0.83 square degrees fields at high galactic latitude in six filters spanning $300-$ $1000 \mathrm{~nm}$ to magnitude limits ranging from 22.1 to 23.8. Newberg et al. (1999, ApJS 123, $377)$ present a catalog containing the measurements of 2262 sources in five Sloan passbands. Color plots of the data show that stars, galaxies, and quasars are fairly well separated by color alone. The stars are compared with synthetic photometry from Kurucz models; the agreement is consistent with the errors in the data.

Mermilliod et al. (1997, A\&AS 124, 349) describe the new version of the General Catalogue of Photometric Data, a most useful database of photoelectric-photometry data that has been updated and completely rebuilt. The Master Index contains information for over 200000 stars and components. Public access to the data is provided through the World Wide Web at http://obswww.unige.ch/gcpd/gcpd.html, and a connection has been established with the ADS Abstract Service which allows to retrieve data directly from the ADS output pages. Hauck \& Mermilliod (1998, A\&AS 129, 431) present a new version of the $u v b y \beta$ catalogue comprising almost 65000 stars and covering the literature to the end of 1996.

Zaritsky et al. (1997, AJ 114, 1002) present first results (1000000 stars) of a digital photometric survey (CCD-UBVI) of the magellanic clouds; they expect to obtain $B, V$ photometry for 25 million stars, and $U$ and $I$ photometry for 10 and 20 million stars, respectively, over the entire survey area. 


\subsection{Atmosphere and sky}

Esslinger \& Edmunds (1998, A\&AS 129, 617) estimate the likely performance of adaptive optics for photometric observations using both real and simulated adaptive-optics data, and provide a useful guide to the feasibility of using adaptive optics in astronomical programmes requiring particular photometric accuracies.

Mattila et al. (1996, A\&AS 119, 153) have measured the sky brightness at ESO, La Silla, during 40 moonless nights in 1978-1988 and the sky brightness variations both during individual nights and from year to year. They found that the long-term variations are well correlated with the solar activity. Chromey et al. (1996, PASP 108, 944) present observations of $B V R I$ surface brightness gradients in the twilight sky, and discuss some strategies for the treatment of gradients in the moonlit and dark sky.

\subsection{Data analysis, image processing, reduction techniques}

Harmanec \& Horn (1998, JAD 4, 5) describe a complete package of programs and data files representing a powerful tool for accurate reductions of photoelectric observations, their transformation to a standard photometric system, archiving and subsequent retrieval. The package (Fortran 77) is especially suited for the reduction of Johnson $U B V$ or Strömgren uvby observations but it can also be adapted to reduction of data obtained in other photometric systems.

Ortiz-Gil et al. (1998, A\&AS 128, 621) describe a new procedure for the reduction of "Carte du Ciel" plates, together with a photometric calibration yielding an accuracy of 0.09 mags.

Nicolet $(1996$, BaltA 5, 417) discusses the evolution of reduction techniques at the Geneva photometric system and offers guidelines to obtain modern magnitudes and color indices that are comparable to the initial data given in the published catalogs.

Naylor (1998, MNRAS 296, 339) presents an optimal extraction algorithm for imaging photometry, and finds a gain of around $10 \%$ in signal-to-noise ratio over normal aperture photometry. Code implementing these algorithms is available at http://www.astro.keele.ac. $\mathrm{uk} / \tilde{\mathrm{timn}} /$. Alard et al. $(1998$, ApJ 503, 325) present a new method designed for optimal subtraction of two images with different seeing and demonstrate that it is possible to derive an optimal solution from a least-squares analysis using all the pixels of both images, and that it is possible to fit the differential background variation at the same time and that PSF variations can be easily handled. Mighell (1999, ADASS 8, 317) describes and reviews basic algorithms for the production of stellar photometry from CCD observations, and discusses in detail some basic techniques of aperture and PSF-fitting CCD stellar photometry. Koranyi et al. $(1998$, PASP 110,1464$)$ describe a robust and computationally-efficient technique for obtaining self-consistent zero points for a set of overlapping photometric and nonphotometric images, a method allowing for calibration of an entire survey from only a few photometric frames-or at least the determination of mutually self-consistent zero points in the absence of any photometric reference.

\subsection{Resolution}

The participants of the conference "Photometric Systems and Standard Stars", held at the Moletai Observatory, urge all stellar photometrists to apply the following principles in their research work:

1. Determine the response functions of your photometric equipment as precisely as possible. The functions should include all components of your photometer: the sensitivity of the detector, transmittance of filters and lenses, reflectivity of telescope mirrors. If magnitudes or color indices are not transformed to outside the atmosphere, the response functions should include transmittance of the atmosphere at the corresponding zenith distance.

2. Make special efforts to match the response functions of your photometric systems to the standard ones. This is especially important working with CCD detectors with different 
response functions in the ultraviolet. Here the filters should be selected for each light detector individually.

3. Use transformation to outside the atmosphere methods which take into account the dependence of the atmospheric extinction coefficient on spectral type, luminosity and interstellar reddening. This is important to all photometric systems with bandwidths $\leq$ $20 \mathrm{~nm}$, especially in the ultraviolet.

4. Use reduction methods to the standard system that take into account nonlinear terms, luminosity and interstellar reddening effects. This is important to all photometric systems (broad, medium and narrow).

5. For obtaining detailed equations for transformation of your instrumental magnitudes and color indices to outside the atmosphere and for reductions to the standard system, use the method of synthetic photometry.

6. A search of the most effective photometric systems for medium band photometry should be continued. We recognize the efficiency of the Vilnius and the Stromvil systems for photometric classification of stars of all spectral types and for identification of peculiar stars of many types, affected by interstellar reddening.

7. The necessity of continuing spectrophotometric observations of stars is confirmed. It is especially important to increase the accuracy of absolute calibration of standard stars and the Sun; this is necessary for a precise comparison of the observed energy distribution functions with the synthetic spectra of model stellar atmospheres and for absolute calibration of photometric systems.

8. For applying the method of synthetic photometry to diverse photometric systems, we need energy distribution functions of stars of different spectral and luminosity classes, metallicities and peculiarity types. However, some types of stars are not sufficiently represented among stars with energy distribution functions available. Such are supergiants of different spectral classes, carbon-rich stars, horizontal-branch stars, pre-main-sequence stars, etc. Spectrophotometry of such stars (including the ultraviolet in the $300-400 \mathrm{~nm}$ range) is of great importance.

9. Further efforts to improve the model stellar atmospheres and their synthetic spectra for various temperatures, gravities and metallicities, necessary for calibration of photometric systems, are appreciated. Special efforts should be directed to improve the theoretical fluxes for supergiants, M-type stars and carbon-rich stars.

10. We recognize the value of small telescopes ( $\leq 1$ meter), coupled with high quality modern instrumentation, for research and for education. They are ideal instruments for calibration of spectrophotometric and photometric observations, for setting up standard stars in different photometric systems (especially for CCD photometry), for survey observations of many kinds, for measurement of the atmospheric extinction necessary to correct observations made with large telescopes, for monitoring variable and suspected variable stars and quasars, for asteroseismological observations of white dwarfs and other related objects, for supernova patrol and for many other tasks.

11. We recognize that light pollution is a growing threat to observational astronomy, and that astronomers should be active in efforts to minimize its negative effects; much effort should be made to improve the quality of nighttime lighting, which will also save energy.

\subsection{Books}

A handbook of infrared astronomy, a very useful book by Ian S. Glass, Cambridge Univ. Press, 1999. The book deals with infrared emission mechanisms, the infrared sky, aspects of infrared photometry and spectroscopy, and infrared astronomical technology. 


\section{POLARIMETRY (J. Landstreet)}

\subsection{Introduction}

Polarimetry continues to grow as a technique that can provide useful information and constraints on the geometrical organization of complex objects; on the nature, location, and sizes of scattering particles; and on magnetic field structures. A number of polarimeters are in active service at observatories such as the Anglo-Australian Observatory (Australia), the Observatoire du Pic-du-Midi (France), the Steward Observatory and the Pine Bluff Observatory (USA), the South African Astronomical Observatory, the Vainu-Bappu Observatory (India), the Crimean Astronomical Observatory and the Special Astrophysical Observatory (Russia), and the University of Western Ontario Observatory (Canada).

Below I offer a sample of some of the polarimetric observing programmes and the corresponding modelling efforts which have recently been reported, as well as references to reviews. This selection is by no means complete, but further work can be found by consulting the references in the papers cited here. The usefulness of such a survey is not nearly as great as it was in past years, now that most working astronomers can access powerful search tools to find articles quickly over the World Wide Web (particularly via the Astrophysics Data System at http://adswww.harvard.edu), but it is hoped that this survey will still be of value to some readers.

\subsection{Clouds in star-forming regions; pre-main-sequence stars}

Polarimetry may provide valuable constraints that help to help understand the geometrical organization of clouds in star-forming regions and of circumstellar material and jets in the vicinity of young stars, since scattering of stellar radiation by irregularly distributed clouds can easily lead to net linear polarization. In some cases polarization by magnetically aligned grains is also implicated. Polarimetry of young objects is increasingly carried out in the IR, and the line between "optical" polarimetry and radio polarimetric observations is becoming more and more blurred. An example of the newly developing continuum linking optical/IR and radio is the polarimetric study of the Orion molecular cloud at $100 \mu \mathrm{m}$ (from the Kuiper Airborne Observatory) and submillimeter regions by Schleuning (1998, ApJ 493, 811).

Young stellar objects have been studied by modelling linear and/or circular polarimetric near IR observations by Chrysostomou et al. (1996, MNRAS 278, 449; 1197, MNRAS 285, 750 ). The second of these papers reports the first imaging measurements of circularly polarized light, which is strong enough to map in the YSO GSS 30.

Polarimetric studies of Herbig Ae/Be stars are ongoing. Close et al. (1997, ApJ 489, 210) report the first adaptive-optics near-IR images of linear polarization of the material around the very young Ae star R Mon, which is obscured by its nebula in visible light but detectable in the IR. Coordinated photometric and polarimetric observations of RR Tau are described and modelled by Rostopchina et al. (1997, A\&A 327, 145). Linear and circular polarization variability in HD 100546 is reported by Clarke et al. (1997, A\&A 347, 590). Variability of the linear polarization of HD 139614 was studied by Yudin et al. (1999, A\&A $345,547)$. Spectropolarimetry around $\mathrm{H} \alpha$ for a number of Herbig Be stars is reported by Oudmaijer \& Drew (1999, MNRAS 305, 166); such data allow one to separate the scattering contributions of electrons and dust.

\subsection{Circumstellar environment of hot stars}

It has been known for more than two decades that the radiation from hot emission line stars is often linearly polarized due to the presence of circumstellar material, either in a massive wind, as occurs in Wolf-Rayet stars, or in material which has to some extent taken up residence around the star, as in Be stars.

Depolarization across the strong emission features of the Wolf-Rayet $+\mathrm{O}$ binary $\gamma^{2}$ Vel has been reported by Eversberg et al. (1998, PASP 110, 753), using a new spectropo- 
larimeter. In contrast, no variation across lines in the WR star HD 165763 is found by Kurosawa et al. (1999, AJ 118, 539), who consider the observed linear polarization to be largely interstellar.

Modelling of Be star envelopes using large data sets that include polarimetry is becoming more common. Quirrenbach et al. (1997, ApJ 479, 477) report such models for seven Be stars; Wood et al. (1997, ApJ 477, 926) discuss a model for $\zeta$ Tau; and Bjorkman et al. (1998, ApJ 509, 904) model UV and visible spectropolarimetry of the B[e] star HD 50138. Other observations of Be stars are reported by McDavid (1999, PASP 111, 494).

\subsection{Polarimetry of magnetic $A$ and $B$ stars}

Polarization measurements, both of single (metallic or Balmer) spectral lines, and of broad spectral bands, play a key role in measurements of stellar magnetic fields. G. Mathys has been carrying out a major programme of spectropolarimetric observations of southern magnetic A and B stars, and has shown that several moments of a general stellar magnetic field are constrained by such data. Some recent field measurements are described by Mathys \& Hubrig (1997, A\&AS 124, 475). Modelling of magnetic field geometries based on a variety of observed magnetic field moments, as well as on the broad-band linear polarization variations found by Leroy, is discussed by Landolfi et al. (1997, A\&A 322, 197).

\subsection{Giant stars}

Because of mass loss, evolved stars sometimes display linear polarization due to scattering from circumstellar material. Strong variability of the optical linear polarization of the RV Tau star AR Pup is reported by Raveendran (1999, MNRAS 303, 595). Non-zero linear polarization was detected in the $\mathrm{mm}$-wave CS $\mathrm{J}=2-1$ line in the evolved stars IRC+10216 and CRL 2688 by Glenn et al. (1997, ApJ 487, L89); these results suggest the presence of deviations from spherical symmetry in the circumstellar envelopes of these stars.

\subsection{Magnetic white dwarfs}

Linear and circular polarimetry, in broad bands and in spectra, continues to be a powerful tool for the study of the large magnetic fields of white dwarf stars. New magnetic white dwarfs are in part identified by searches for broad-band circular polarization. Once identified, spectro-polarimetry provides a powerful tool to assist in modelling the magnetic field strength and structure found on individual stars.

Recent work in this field includes modelling of spectra of white dwarfs with fields in the range of $10^{6}$ to $3 \times 10^{7} \mathrm{G}$ (Friedrich et al. 1996, A\&A 309, 227), a search for kilogauss-level magnetic fields in pulsating white dwarfs by Schmidt \& Grauer (1997, ApJ 488, 827), further observations of the very peculiar magnetic emission line white dwarf GD356 (Ferrario et al. 1997, MNRAS 289, 105), and a study of the cool white dwarf LHS 2229 which appears to show bands of a C-H compound in a field of about $10^{8} \mathrm{G}$ (Schmidt et al. 1999, ApJ 512, 916).

\subsection{Cataclysmic binaries and AM Her systems}

Close binary systems containing a magnetic white dwarf, often with mass transfer onto the degenerate star, are being identified in ever greater numbers. In such systems the magnetic field of the white dwarf is often revealed by strong linear and/or circular polarization of continuum light.

A search for variability in the broad-band linear polarization of several non-magnetic cataclysmic variables by Naylor et al. (1996, MNRAS 282,873 ) has resulted in quite tight upper limits, indicating that intrinsic polarization in these objects is small.

Optical polarization data are used to model the accretion region of the AM Her system RE J1844-74 by Ramsay et al. (1996, MNRAS 282, 726). Circular polarization is detected 
in the proposed AM Her system EXO 032957-2626.9 by Cropper (1997, MNRAS 289, 21), confirming that this object is a member of the class. Some theoretical models of the polarized emission from such objects are described by Rashed (1997, ApJ 484, 341).

Linear polarization oscillations in the DQ Her star PQ Gem are reported by Somov et al. (1998, A\&A 335, 583).

\subsection{Books}

An excellent new book on polarimetry has recently been published by Jean-Louis Leroy. The french edition, La polarisation de la lumière et l'observation astronomique, appeared in 1998 under the imprint of Gordon \& Breach Science Publishers. An English version should soon be available as well, from the same publishing house. This compact monograph surveys the entire subject of astronomical polarisation from planetary surfaces and atmospheres, through stars, to extra-galactic sources. It is written at a level which is suitable for a range of readers, from students and amateur astronomers to professionals, who want a clear general introduction to the subject.

Christiaan Sterken President of the Commission 